\title{
Constructive activism in the dark web: cryptomarkets and illicit drugs in the digital 'demimonde'
}

Alexia Maddox, National Drug Research Institute, Curtin University, Melbourne Australia, alexia.maddox@curtin.edu.au, with correspondence to go to a.maddox@deakin.edu.au (corresponding author).

Dr Maddox was a research officer at the National Drug Research Institute, through which the research was conducted. She also works as a research officer at Deakin University.

Monica J. Barratt, ${ }^{a}$ Drug Policy Modelling Program, National Drug and Alcohol Research Centre, UNSW Australia, Sydney NSW 2052, Australia, m.barratt@unsw.edu.au

${ }^{\mathrm{b}}$ National Drug Research Institute, Faculty of Health Sciences, Curtin University, GPO Box U1987, Perth WA 6845, Australia.

${ }^{\mathrm{c}}$ Centre of Population Health, Burnet Institute, 85 Commercial Road, Melbourne Vic 3004, Australia.

Dr Barratt is an NHMRC Postdoctoral Fellow conducting research into the social and public health implications of internet technologies for people who use illicit and emerging psychoactive drugs.

Matthew Allen, School of Communication and Creative Arts, Deakin University, Melbourne, Australia, matthew.allen@deakin.edu.au

Professor Allen is Head of School and Professor of Internet Studies in the School of Communciation \& Creative Arts at Deakin University.

Simon Lenton, National Drug Research Institute, Curtin University, Perth, Australia, S.Lenton@curtin.edu.au 
Professor Lenton is a Deputy Director at the National Drug Research Institute and a clinical psychologist in private practice.

Post-print version: Date 8/09/15

Final version of the article published as follows:

Maddox, A., Barratt, M. J., Allen, M., \& Lenton, S. (2015). Constructive activism in the dark web: cryptomarkets and illicit drugs in the digital 'demimonde'. Information, Communication \& Society, 1-16. doi: 10.1080/1369118x.2015.1093531

http://www.tandfonline.com/doi/abs/10.1080/1369118X.2015.1093531 


\section{Constructive activism in the dark web: cryptomarkets and illicit drugs in the digital 'demimonde'}

This paper explores activism enacted through Silk Road, a now defunct cryptomarket where illicit drugs were sold in the dark web. Drawing on a digital ethnography of Silk Road, we develop the notion of constructive activism to extend the lexicon of concepts available to discuss forms of online activism. Monitoring of the cryptomarket took place between June 2011 and its closure in October 2013. Just before and after the closure of the marketplace we conducted anonymous online interviews with 17 people who reported buying drugs on Silk Road (1.0). These interviews were conducted synchronously and interactively through encrypted instant messaging. Participants discussed harnessing and developing the technological tools needed to access Silk Road and engage within the Silk Road community. For participants Silk Road was not just a market for trading drugs: it facilitated a shared experience of personal freedom within a libertarian philosophical framework, where open discussions about stigmatised behaviours were encouraged and supported. Tensions between public activism against drug prohibition and the need to hide one's identity as a drug user from public scrutiny were partially resolved through community actions that internalised these politics, rather than engaging in forms of online activism that are intended to have real-world political effects. Most aptly described through van de Sande's (2015) concept of prefigurative politics, they sought to transform their values into built environments that were designed to socially engineer a more permissive digital reality, which we refer to as constructive activism.

Keywords: dark web, online social activism, online community, digital ethnography, e-Commerce, illicit drugs

\section{Introduction}

This paper is an ethnographic exploration of the original Silk Road, an online cryptomarket and associated community, from which we develop the notion of constructive activism. Cryptomarkets are a digital demimonde, a marginalised world that is an isolated part of everyday lives, operating both on the fringes of the 
publically accessible Internet (through Tor) and social mores. We argue that the online community of Silk Road enacts social resistance by using online technology to build an alternative reality that enables peer-to-peer trading of drugs and associated discussions of drug-taking. This form of resistance is different from norms of online activism intended explicitly to realise broader political effects outside of their specific online space. Such resistance has not yet been acknowledged within the notion of connective action (Bennett \& Segerberg, 2012), the dominant paradigm for analysing online activism. In this paper, we seek to extend understanding of online activism, using the lens of prefigurative politics (van de Sande, 2015). Through this lens we aim to explore how permissive spaces online, not yet in existence in the physical world, enable radical politics through Internet techno-culture, even if their activities are largely invisible to the world.

Silk Road, a cryptomarket for illegal drug trading, was launched in February 2011 and became more publically visible from 2012 until its closure in 2013. Cryptomarkets are digital platforms that facilitate $\mathrm{P} 2 \mathrm{P}$ trade of goods and services with the added features of recommendations systems and a reliance on cryptocurrencies such as Bitcoin. They utilise anonymising Internet routing technologies (principally Tor, short for The Onion Router) to conceal the physical-word identity and location of users and create an open network for such interactions (Cardullo, 2015, p. 8). Tor is the backbone of the dark web, which Gehl $(2014$, p. 2) describes as a part of the Internet that cannot be accessed by mainstream software. We argue that most people go to the dark web to 'be hidden' and that they are not seeking a political voice, in the mainstream political sense, because they believe that their activity will be prosecuted in other spaces rather than contributing to social change. Silk Road was representative of the alternative and decentralized economies made possible when hidden in the dark 
web.

Recent research has begun to reveal the nature of cryptomarkets and the people who use them to access drugs (Aldridge \& Décary-Hétu, 2014; Barratt, 2012; Barratt, Ferris, \& Winstock, 2014; Christin, 2013; Martin, 2014; van Hout \& Bingham, 2013a, 2013b, 2014). Barratt, Ferris, et al. (2014) found that people using cryptomarkets believed the markets provided access to better-quality drugs, more conveniently, and with access to a vendor-rating system to increase confidence in supply. But such markets are not just about transactions. van Hout \& Bingham (2013a, 2013b, 2014) found that there was an active community operating inside cryptomarkets, increasing opportunities for drug discussion online. Such drug-use communities already operate in the clear web, described by Barratt, Allen and Lenton (2014) as places where drugusing peers developed counterpublics where dominant discourses of drug use being inherently problematic were resisted and alternative discourses (safety; pleasure) were emphasised. However increased global Internet surveillance and censorship, and the use of corporately regulated social networking sites such as Facebook have reduced the availability or freedom to use online spaces to enable such counterpublics (Barratt, Lenton, \& Allen, 2013). Drug-use communities are increasingly utilising the hidden spaces of the dark web, alongside the trading environment for illegal drugs.

The founder of Silk Road claimed it was an economic experiment 'giving people the freedom to make their own choices, pursue their own happiness, how they saw individually fit' (Ulbricht, 2015). The libertarian ideology expressed in this statement and afforded technologically by the Silk Road platform resonates with traditional beliefs about the purpose and meaning of the Internet to change society through radical reconfiguring of the relations of power, information and exchange dating back 
many years. We need to understand how Silk Road operated not just as a drug-trading market but as a political act and environment. We shall begin, therefore, by reviewing literature on existing forms of online social activism and then use the case study of the Silk Road community to show how connective activities online are preconfigured, reconfigured, and channelled into constructive activism.

\section{Prefigurative politics and constructive action}

It is broadly agreed that, from a social movement perspective, the Internet helps circumvent state-sanctioned silencing of marginalised voices and the denial of conversational opportunities for alternative social norms (Garrett, 2006, p. 203). For drug-use communities the Internet allows experiential discourses to develop contrary to the hegemonic narrative of illicit drug use as inherently problematic (Barratt, Allen, et al., 2014). However the Internet releases two opposing possibilities, both towards emancipation and towards domination (Manion \& Goodrum, 2000, p.18). As Garrett (2006, p. 210) observes, while digital networked technologies afford new forms of social resistance and collective action, contentious activity online also creates new opportunities for its demobilization by elites who control the infrastructures. Specifically, Garrett notes that when a particular use becomes too threatening to social norms, challengers may be denied access to resources, or a systems architecture may be modified to prevent undesirable uses. In response acts of resistance to this very act of technological domination appear, involving creating alternative digital locations through code development (McInerney, 2009), file-sharing practices (Andersson, 2011) or disrupting existing locations associated with elites, (cf. Coleman, 2010, 2012, 2014; Coleman \& Golub, 2008). These forms of engagement are not commonly considered to be explicit forms of online social activism, perhaps 
because such activism is construed to be using the Internet against a non-networked elite whereas, more and more, social resistance must accommodate the sophisticated domination of network spaces by governments, corporations and other elite entities.

In proposing a specific theory of social activism online, Bennett and Segerberg (2012) revoke the notion that collective identity is the cohesive source of a social movement (Gerbaudo \& Treré, 2015), shifting the focus of activism to the public spectacle resulting from increasingly personalised political participation through sharing on social media. They argue that the individual contribution is the starting point of connective action through self-motivated sharing of personalized ideas, plans, images and resources with networks of others (p.753). Communication technologies create digital traces of connective action that serve as a memory record or 'action repertoires', supporting the growth and stabilisation of network structures (pp.753-755). Whilst Bennett and Segerberg's (2012) notion of connective activism speaks strongly to the diversity of views and modes of online interaction and mobilisation within a community, it does not encompass the historical trajectory of Internet user-cultures that reject or ignore engagement with existing governance structures in favour of developing new and permissive digital frontiers through collaboration and code.

van de Sande (2015) introduces the idea of prefigurative politics to conceptualise how communities use communication technologies and code construction as a form of online activism. Drawing on theories of socialism, anarchism and Graeber's analysis of the Occupy movement, van de Sande argues that online social movements founded upon radical politics may, quite deliberately, have little impact upon existing political structure because they do not aim to reconcile 
with the dominant political and economic order. Rather they create the society that they idealise 'in miniature' (p.178), prefiguring the 'world to come ....in the here and now' (p.189), building a new society within the shell of the old (p.191). This prefigurative action is more possible through the powerful opportunities to construct alternative worlds in code, online: the construction of the world-in-miniature is as important as its occupancy.

Cryptomarkets, such as Silk Road, can therefore be seen as a form of constructive activism that uses prefigurative politics to construct a world that is 'not yet' and through its existence seeks to 'alter the world, not only to construct a different reality, but to simultaneously confront its old forms' (van de Sande, 2015, p. 191). This paper will draw on the experiences of a small sample of drug users who participated in Silk Road 1.0, who were interviewed through encrypted instant messaging, to explore how this world was constructed as a space of activism. A reasonable proxy estimate of the membership base for the Silk Road community can be found in the Federal Bureau of Investigation's (FBI, 2013) indictment of Silk Road's founder, with Silk Road's marketplace reported to have 147,000 active buyer accounts by July 2013. Silk Road was the natural choice as a site of study, due to its dominant position in the marketplace (the FBI reported its sales volume as 9.5 million bitcoin) and the relatively high level of community trust in its leadership compared with more recent cryptomarkets. Drawing on the lens of prefigurative politics, this paper will discuss how the combination of radical politics, such as libertarianism, and the presence of community collaboration resulted in the development of frontier digital spaces. 


\section{Methodology}

We conducted a multi-sited, digital ethnography during most of the lifecycle of Silk Road, monitoring the marketplace and associated forums from June 2011 to its closure in late 2013 and beyond. The study was approved by the Curtin Human Research Ethics Committee (HR 26/2012). Further analysis of the methodological and ethical issues arising from this study may be found in Barratt \& Maddox (forthcoming). Our study preparation included the development of a security protocol to ensure anonymity of our participants, practice and mastery of these technical skills, and amendments to our methodology to match changes in the technological environment of the cryptomarket. Participants were specifically informed that they should refrain from providing any detailed information about criminal activities.

Maddox (Author 1) conducted 17 encrypted online interviews between September 2013 and June 2014, with all but one interview conducted after the closure of Silk Road. Recruitment of participants, who were aged 18 years or over and reported buying drugs at least once from Silk Road, occurred through Twitter, Reddit and a dark web forum and chat channel. Interviews took 3 hours to complete on average, ranging from 2 to 4 hours. No monetary compensation was offered. A participant information sheet was available from the project website, and the interviewer, Author 1 obtained text-based consent to participate after provision of the link to this document and a short summary of its contents. An encrypted channel was formed between Author 1 and the interviewee, and the interviewee was also able to verify Author 1's identity by matching her public key, also published on the project website, with her cryptographic fingerprint in the interview. The interview was unstructured with Author 1 using a series of prompts to guide the conversation through topics of interest, including: a description of participants' engagement with 
Silk Road from first discovery of until closure; their drug use trajectories; their computer and Internet use histories; how they dealt with Silk Road's closure; and their experience with other cryptomarkets. Throughout this process, Author 1 jotted information onto a printed life timeline template, and used this document to prompt further probing of the nature and dependencies of events in the interviewee's life.

After each interview was complete, Author 1 de-identified the text log before saving the data. A participant I.D. number was assigned. Interview transcripts were entered into NVivo. During first pass coding, Author 1 conducted open coding of content into in vivo nodes under predetermined node trees. A second pass through each interview transcript was completed to apply these new nodes more consistently. To assist with validity and transparency of coding, Barratt (Author 2) selected 2 transcripts using a random number generator to code independently using the same node structure. We calculated kappa values for coding similarity for these two example cases. According to McHugh (2012), kappa values of 0.6 or above indicate at least moderate agreement between coders. One quarter (28\%) of nodes had kappa values below 0.6 , and were therefore subjected to further scrutiny where discrepancies between coders were resolved. Following this process, Author 1 conducted a final pass over the dataset applying the coding structure assisted by refined code definitions.

\section{A description of the interview cohort}

Of the 17 participants in the research, 15 reported as male, one as 'nonbinary', with one was unknown. The majority of participants (10) reported being under 25 years of age, with three of these reporting to be under 20 . Three participants reported to be between 30 and 40 age bracket and one over 50, presenting both seasoned and reconnecting behaviours to drug use and drug use cultures. Three 
participants did not report their age. Three participants reported not finishing secondary education, four had and six reported engagement in or completion of tertiary education. Three participants reported being students, four participants reported being IT professionals, four participants reported working in the service sector, one in the media, one in education and one retired.

In terms of country of origin and Internet access, five participants reported living in the USA, 4 in Australia, one in Sweden and one in the UK. This geographic profile suggests that Internet access was relatively available for all participants. Within the cohort, a smaller group of participants identified as native to what might be termed digital cultures such as gamers (1), cypherpunks, cryptonerds and phreakers (4) and cyber-libertarians (7). In terms of community participation profile participants identified as community insiders (2), active forums contributors (7), active listeners on forums (3) and disengaged drug purchasers (4). Four participants reported being actively oriented to cryptomarkets as a social movement and activist space, whilst most participants reported to hold liberal values and preferred purchasing their drugs in these online spaces over place-based options, relating this choice to their personal sovereignty and freedom.

Participants reported engaging first with Silk Road across a wide range: from being present at its launch as a beta tester to being first there a few weeks before its closure. On average, participants were active in the Silk Road marketplace for just under a year: some participants actively used the market place at regular intervals, whilst others browsed and purchased infrequently suggesting variability in exposure to the marketplace. 
This cohort of participants present diverse perspectives, but are not representative of the Silk Road community as a whole. Participants who did not use drugs but engaged with the cryptomarket communities for other reasons were not a part of this research due the goals of the project in engaging with people who had purchased drugs on Silk Road. Through the ethnographic monitoring and research presence in these environments over time, it was clear that there was a greater diversity of people and activist perspectives than represented in the dataset. Other limitations to the study include those associated with self-report data and our inability to verify the claims made by anonymous participants about their cryptomarket activities. However, the experiences presented here point to evolving forms of online activism that reveal prefigurative politics in action; that is, the presence of radical politics, disengagement with the establishment and internalised social activism, through a combination of identity compartmentalisation and community collaboration, that focused upon building new worlds through code.

\section{Entering the digital 'demimonde'}

During the interview P18 referred to scoring drugs through place-based and face-to-face transactions as entering the demimonde.

1:28:11 pm [P18]:[...] But risk? sometimes i do miss the kind of entering the "demimonde" aspect of scoring drugs, trawling the seedy underbelly for gear and the danger and whatnot - but it's like reminiscing about your uni ddays and laughing about how poor $\mathrm{u}$ were $[\ldots]$

Entering the Silk Road can be likened to entering a digital demimonde in the underworld of the dark web. Using Silk Road involves a specific set of skills and 
knowledge to access the dark web, principally to access Tor, to enact specific personal security practices through the use of encryption and anonymising technologies, and to access and transact with a cryptocurrency, Bitcoin. Participants who had been active on Silk Road since its launch reported that there was initially a high level of technical skill needed, which effectively limited access to the site and the community, creating a small techno-elite who in their shared interest in such sophisticated computer use became a unique and supportive community culture. However, as the environment and access to Bitcoin became more user friendly, participants reported a broader demographic of users, 'the masses' and 'n00bs' with some participants representing this more recent influx of users who had not been members of the smaller group. P14 explained the typical hurdles a user to needed to overcome to access the marketplace and making a purchase.

4:38:31 pm [P14]: The darkweb is unique in that it isn't particularly userfriendly. Techologies like facebook and twitter might take five seconds to set up an account and then you're able to utilize all of the functions of the site. With SR and other darknet marketplaces it isn't that straightfoward. You have to learn how to encrypt and decrypt messages, and how acquire a relatively esoteric virtual currency. And of course these places aren't well advertised, beside being demonized in the news-media.

Regardless of their range in skill level, participants found their way into Silk Road through various entry points. The majority (10) learned about it through their social networks, and the remainder read about it in Gawker magazine (Chen, 2011), discussions in online forums, Reddit, and the Tor wiki. P20 describes his initial impressions upon moving into this space.

7:33:33 am [P20]: First impressions was that it was pretty exciting, it's pretty weird to look at and be like aware that you can just circumvent the law 
anonymously in such an ingenious fashion (tor+bitcoin, or encryption: edward snowden's ${ }^{1}$ "modern defense against the dark arts class")

For some participants, engaging with Silk Road was not solely (or even primarily) about the drugs themselves but about being in a space that permitted personal freedoms where illicit drug use was not stigmatised. As P18 found upon moving into this space, there was a thriving community culture that expressed itself through the forums associated with the Silk Road marketplace and an adoration for the forum administrator, the Dread Pirate Roberts (DPR). As a techno-luddite, he described trampling all over the norms and expectations of the space and confessed to making a lot of 'n00b' mistakes as he got to know the place.

12:13:21 pm [P18]: I didn't participate on the sr1 forums at all. The rule that you must spam 50 posts in the newbie section before $u$ can post (the coachella directive) was daunting and I was struggling to keep up with it all just observing and marvelling at it and going thru the archives. The flame wars and the arguments (particularly in the meth vendors thread where the posters have endless stamina) was fascinating. [...] But I was amazed at the forums and the cult-like adoration of DPR. He would occasionally post in the forums, like Moses or the pope coming out on the balcony in St Peter's square to address his loyal subjects.

It was commonly described by our participants that entering Silk Road felt like an amazing cornucopia of people and drug diversity that generated a sense of wonderment and disbelief.

\footnotetext{
${ }^{1}$ Edward Snowden famously disclosed extensive information about the practices of the US National Security Agency (NSA) with regard to PRISM and other US surveillance programs and confirmed the large-scale mass surveillance of the telecommunication and electronic messages of governments, companies, citizens. For further discussion of the implications his disclosure see Bauman et al. (2014)
} 


\section{Community, co-operation and paradigms of personal freedom}

Participants reported observing an active community in the Silk Road forums, where there was co-operation, collaboration and the assertion of personal freedoms based upon a mix of cyber-libertarian philosophy and liberal values. Some participated in the forums to research vendors, to read or write trip reports, to learn about the drugs they wanted or to be updated on the latest security practices for making anonymous drug purchases. P20 discussed his experience of engaging in the forums as impeded by the technology (Tor) but that this didn't stop him perusing the cultural elements of the site:

7:57:12 am [Interviewer]: I'd like to talk a bit about SR again. Can you tell me a little about the forums (once you found them). Did you lurk? engage? 7:59:17 am [P20]: mostly lurked, the slowness of Tor makes it pretty difficult to successfully submit posts on a forum without multiple attempts, but I'd sometimes post on a thread concerning a vendor I'd bought from etc. Also there were some interesting sections like on Philosophy, Economics, etc. and one called "DPR's Book Club" which explored many texts such as libertarian stuff like Rothbard

Participants not only had the opportunity to learn about and discuss libertarian philosophy, they also had the capacity to contribute and receive in the space, in perhaps the ultimate act of prosumption. Prosumption simultaneously involves the acts of production and consumption, particularly through user generated content and peer production online in ways that problematise the distinction between the producer and the consumer (Beer \& Burrows, 2010; Ritzer \& Jurgenson, 2010). P15 describes how he gives and gains through his forum contributions and trip reports reviewing drugs he's consumed from the vendors on the site. Vendors would offer free samples for product reviews. 
9:04:34 am [P15]: Generally they would start a thread on the forums, and it was a first-come, first-serve basis for established members. Usually people with a decent amount of forum posts/presence, and good buyer stats 9:04:58 am [interviewer]: the kind of people likely to post a review and give back?

9:05:47 am [P15]: Exactly. And of course, if you can subtley demonstrate your prowess at writing in your post in the thread, that helps too ;)

$[\ldots]$

9:07:36 am [P15]: The best way to get someone to do work is to make them think it isn't work. Haha

9:07:50 am [P15]: People aren't going to say "Oh man, I have to sample these drugs today. Bummer"

The forum spaces were a place for people to be open about their drug use and share their interests, both discussing and enacting values around personal freedoms. For participants who were not able to discuss their drug-use experiences through their in-person networks, either due to fear of social stigma or not having access to likeminded friends, the Silk Road community was a place where they could openly engage. For P14, Silk Road was a means to an end 'Buy drugs, log off.', as he thought he already had sufficient people to talk to about his interests (cryptography) and drug preferences (LSD), seeing no need to do it with anonymous individuals online. Nevertheless, he observed a sense of community in the forums. P12 enjoyed being able to share his experiences online with other drug users, where he used the Silk Road forums to 'discuss, ask questions, and talk about experiences'. P1 discussed using the forums for security tips and cryptography discussions, highlighting the presence of trust and his sense of the connection between the people in the space.

7:49:07 PM [P1]: [...] There's a security thread with stickies about 'how to use sr totally anonymously' and another for the cryptography... that's a good point to make, and I implied it earlier. I trust sr. The people that use it are smart. And most are there to cooperate and help one another. 
Similarly, P19 observed that the early Silk Road community was characterised by exclusivity, contribution and radical politics.

2:31:44 pm [P19]: Most people wanted to help and contribute in SR 1.0. It was exclusive, not many knew how to get to it, it was revolutionary, and practically anarchic.

As a space of radical politics $\mathrm{P} 15$ highlights the activist mentality of the space and its desire to disrupt the status quo. When talking about the slight arrogance that arose amongst the Silk Road community from lasting 2 plus years, with 'the entire might of the US government to oppose them', P15 was asked whether this made the community come closer together to support each other whilst 'under attack'.

8:33:18 am [P15]: Definitely. It was a classic us-against-them scenario so we all felt like brothers in arms I suppose.

Idealistically, the site stood as a demonstration that drugs, like all other commercial items, can be traded non-violently while also not putting more money back into gangs and cartels.

For most participants, their engagement with Silk Road operationalised a sense of freedom to consume their drug of choice in the context of doing no harm to others, aligning participants with the cyber-libertarian philosophy of DPR, the administrator of Silk Road. P5 exemplified the drug-use narrative pervasive in the dataset:

7:32:38 pm [P5]: I agree with the idea that information and by association chemicals want to be free. People want to be able to believe and use their body how they choose. I think that we should be able to as gay, drug using, atheistic, and hedonistic and [as] we want. As long as it harms no one else I don't care.

Identifying as a libertarian socialist, P14 outlined the aspects of personal liberty that he sees as essential, including 'Free association, freedom to consume what you with [want], freedom to produce what you wish. All with the condition that your actions 
aren't harming others.' This focus on non-violent personal freedoms was pervasive in participants' perspectives, however P14 acknowledged that the line between harmful and non-harmful behaviour remained indistinct. When asked where he saw the line between what was and was not acceptable, P14 acknowledged that:

4:23:55 pm [P14]: Whether what you are doing is harming others. Trying to define what is harmful is more difficult, and for me that's where the line becomes blurred.

For P11, the freedom to consume was a 'vulnerable type of freedom' given that it was possible to 'buy what you like but not really being able to decide what you like', suggesting that the open market place and unregulated access to drugs was a form of personal freedom through diversity of choice. Initially, this freedom to consume was the appeal for him, but, as he became more familiar with Silk Road, the anonymity itself became the freedom. For P19 the freedom to consume was linked to the capacity to be responsible for one's own choices: 'Responsibility for what you order, receive and use. You have the choice, nobody else. I prefer having the choice.' The sense of 'moral choice' was also articulated by P20. He stated that 'More accurately I could probably say that the concept of getting drugs in general is consistent with a libertarian moral philosophy that acknowledges that just because something is illegal doesn't necessarily make in [it] unethical'.

Similarly, P1 thought that the creator of Silk Road had a moral fight in mind and that his agenda was to point out something that is wrong in this world, the way drug users are 'looked down on' and that Silk Road was representative of a 'group of people that think alike about the world'. He thought that its members were a group of people who 'refuse to accept being a part of a system that is detrimental', observing that these people were more likely to experiment with drugs. P14, citing authors such 
as Orwell, Chomsky, Emma Goldman, and Bakunin, expressed a deep distrust of the state, suggesting that Silk Road was juxtaposed to the state and thus an activist space. P20 overtly made this connection between Silk Road as a libertarian anarchic social model and its opposition to state control.

8:21:00 am [P20]: I believe that libertarian philosophy, which is fundamentally anarchist at its core, anarchist in the sense that all of us are equal moral agents from an existentialist framework

8:21:15 am [P20]: is a core component of dnms [dark net markets] like SR 8:21:48 am [P20]: because they're based on the concept that you have selfownership, so you should be free to legally interact 8:21:55 am [P20]: or rather freely interact 8:22:00 am [P20]: extraneous of the law 8:22:05 am [P20]: which isn't to say an anarchist system lacks law 8:22:30 am [P20]: but rather law that's imposed by the illegitimate use of force, a monopoly on force currently granted to the state 8:22:46 am [P20]: bitcoin is relevant too, that's fundamentally libertarian and inextricable from $\mathrm{sr}$ 8:23:03 am [P20]: if one has a right to their property they also have a right to trade whatever so long as it's voluntary in my view 8:23:10 am [P20]: no victim, no crime

But not all participants thought that personal freedoms in drug choice should exist beyond the administration of the state. Notably, P25 indicated that whilst he identified with libertarian values, he thought that 'drugs should be legalised and regulated by the government, not in a black market, were [where] money is the driving factor and not health the driving factor'.

While the activist mentality appeared to drive collaborative construction through engagement with and maintenance of Silk Road, this attitude appeared more predominant early in the site's existence. The sense of a moral fight and a desire for personal freedoms were representative of some participants' experiences of Silk Road 
in its early years, but the culture of the site was reported to change as more people began to use it, with a different expectation of what it meant to be involved. P15, a late-comer to Silk Road observed this change.

\author{
8:54:43 am [P15]: I was told that the "original" Dread Pirate Roberts (or \\ previous. Who knows how many there have been) was aggressively \\ libertarian/anarchist. By the time I got there, there was still a sense of that from \\ the site as a whole, but it was more reserved. Harm reduction was first and \\ foremost though, and that's always been my biggest focus. You can't stop people \\ from doing drugs (not that I'm implying drug use is inherently immoral/bad), but \\ you can definitely keep them healthy and safe \\ 8:55:18 am [P15]: Healthier and safer, anyways
}

In reflecting on the change in the social dynamics of the space, P15 thought that the site's expansion was now in contradiction to its ideals, noting that 'if a demonstration of political dissent through illegal activity is what one is going for, it's best to keep the community as a group of tight-knit, smart, and cautious people.' P23 also observed the change of culture in the space, from his early experiences in engaging with the site in early 2012 to being active at its close.

9:37:32 pm [Interviewer]: did the feel of the space change much over the time you were active on it?

9:37:50 pm [P23]: yeah

9:37:54 pm [P23]: definitely as it grew

9:37:59 pm [P23]: i mean when i started

9:38:07 pm [P23]: it was almost exclusively cryptonerds

9:38:24 pm [P23]: and people who really used the internet

9:38:26 pm [P23]: over time

9:38:30 pm [P23]: as it got more popular

9:38:33 pm [P23]: everyone was on

9:38:39 pm [P23]: you would have threads like

9:39:02 pm [P23]: 'cen sum1 pls tel me wer to get crck 2dai to 14 terra st, etc etc etc 
9:39:07 pm [P23]: like very unwise people

9:39:16 pm [P23]: also the bigger it got the more undercover agents

9:39:22 pm [P23]: and that really killed the buzz

From the way participants reported growth in their awareness of the meaning of using Silk Road, we can see that the explicit establishment of the Silk Road upon libertarian ideals had the capacity to enact a space for the experience of personal freedom and then develop consciousness about the meaning of freedom. The anonymous interactions that were considered a social norm for the Silk Road community facilitated an openness for discussion of illicit behaviour that many participants were denied outside of its confines, suggesting that its encoded walls promoted freedom rather than constrained it. This possibility of open discussion contributed to the political sensibilities which accompanied interaction with Silk Road.

\section{Compartmentalisation, constructive activism and continuity}

Most participants described keeping their activities on the dark web separate from those of their 'everyday' lives. For example, they rarely revealed their drug user identity and preference of purchasing drugs in an unregulated online marketplace to people that they knew, particularly their work place or their family. P5 reported that the combination of his illicit drug use and engagement with cryptomarkets encouraged him to disconnect these activities from his family and friends due to fears of social stigma and personal security concerns.

7:05:46 pm [Interviewer]: [...] How have your relationships (close ones and friends) flowed with you drug use? Any positives or negatives?

7:07:15 pm [P5]: So much closer with my group of friends of about 5. Slightly alienated from others but still very popular. I can't tell if I think I'm alienated or I am. I find it hard to relate to people sometimes. 
I'm closer and further from my family. Hard to explain.

7:08:25 pm [Interviewer]: sounds pretty natural hey. When you started with Silk Road, did you share that you were doing this with your close friends or keep it just to your friend who you went into this with?

7:09:30 pm [P5]: I honestly told way too many people. I was retarded. My close 5 friends knew and then like probably 10 more. I pretended to stop after a couple months so that those 10 would think I was done.

7:10:12 pm [Interviewer]: yeah - I imagine that could contribute to a sense of risk as you started buying more online. Did your approach work?

7:10:43 pm [P5]: yep.

Several participants reported that the normative social stigma attached to illicit drug use held them back from revealing their behaviours to their family and some of their social networks. Furthermore, the illegal nature of their purchasing and consuming illicit drugs encouraged them to compartmentalise their cryptomarket activities from their everyday lives in order to reduce their risks. However, the Silk Road forum, the associated subreddit and Internet Relay Chat (IRC) channels ${ }^{2}$ were places where participants engaged in these discussions anonymously and in the open (ie publically viewable spaces accessible to most people). P21 described taking drugs as an asocial or private activity, which he was able to share through the Silk Road forums and associated anonymous channels.

6:36:42 pm [P21]: yeah i keep most of my drug use to myself irl [in real life]

6:36:58 pm [P21]: but i'm pretty open about it in the chat and on the forums

This statement reveals one identity but two modes of engagement, aligning with Hine's (2000) notion of a single identity across the online and offline environments. This division of engagement effectively limited the participants' capacity for real-

\footnotetext{
${ }^{2}$ Open online discussion technologies
} 
world activism tied to their everyday lives. Whilst some participants professed to be activists protesting against drug prohibition and discussed alternative forms of regulation during the interview, others preferred to make their contributions anonymously online. For example, P24 discussed being a 'beta tester' for the Silk Road cryptomarket at its outset, looking for security risks and ways that the site may be hacked by competitors or law enforcement. P17, a cyber-security professional, who was not particularly interested in socialising online, admitted to being drawn into discussions of security protocols and encryption practices used in cryptomarkets based upon his personal and professional interests.

3:31:50 pm [interviewer]: Given this [the closure of Silk Road], what are you doing now? Do you continue to purchase from other market places or have you found a suitable alternative approach?

3:34:16 pm [P17]: I have found the one I thought had the best security model, and have championed their cause. I have met others like me who are interested in applying known solutions to the issue of trust to the underground markets.

Similarly, towards the close of each interview, participants discussed their reactions and actions when Silk Road was closed by law enforcement in October 2013, the first significant act of state disruption of the cryptomarket environment. Most participants reported reduction in trust in the online environment, and a sense of loss for the political vision of a different future. The dispersal of the community that resulted from the seizure of the site, however, did not reduce or resolve its members' desire for their personal freedoms. P22 thought that, regardless of the loss of Silk Road, the community connections remained, the values re-emerged and the future began to be constructed in the minds of participants who cared more for their community and continued access to illicit drugs than for the loss of a single site. 
1:41:00 pm [P22]: Well

1:41:07 pm [P22]: It was very different from nowadays.

$[\ldots]$

1:42:47 pm [P22]: People had a lot more optimism and faith in the community back then.

$[\ldots]$

1:45:40 pm [interviewer]: what interests me, is that there remains a "collective" at all in light $f$ events since SR 1.0 was seized.

1:46:24 pm [P22]: Me too

1:46:56 pm [interviewer]: it tells me that there is much more to people's

experiences than just buying drugs

1:47:02 pm [P22]: And not to shameless reference [anonymised], but my

intention with creating [an online forum for the discussion of cryptomarkets

within Tor] is to try and keep that collective intact.

1:47:10 pm [P22]: Yes, agreed.

1:47:30 pm [P22]: Some of the people I have met here are better people than I have met anywhere else.

Participants' reflections on the effects of the closure of Silk Road, both personally and for the community as a whole, demonstrate the fragility (but also recuperative) capacities of the Internet and the social resilience within the environment. These capacities were articulated in the way that Silk Road's closure both threatened participants' trust in the possibility of freedom, yet reinforced their desire to reconstruct that possibility, in other dark-web places.

\section{Conclusions}

What conclusions can we draw from the deep discussion of Silk Road with some of its most articulate participants? First, it is clear that Silk Road was not just a space for trading illicit drugs. Explicitly established with libertarian ideals, its capacity to engender a space for first an experience and then a shared understanding of personal freedom can be seen in the way participants' growing awareness of how 
Silk Road manifested these ideals. Second, Silk Road provided, through anonymity, an openness for discussion of illicit behaviour that many participants were denied outside of its confines. Silk Road's encoded walls promoted freedom rather than constrained it. This possibility of open discussion contributed to the political sensibilities that accompanied interaction with Silk Road. Third, the fragility (but also recuperative) capacities of the Internet were demonstrated in the way that Silk Road's closure both threatened participants' trust in the possibility of freedom, yet reinforced their desire to reconstruct that possibility in other dark-web places.

The strong division within the membership of Silk Road community, observed through the ethnographic monitoring and reported by participants, was between public activism against drug prohibition and the hidden development of permissive spaces within the dark web for drug trading and the sharing of drug information. Additionally the practice of separating their engagement with cryptomarkets and illicit drug use from cryptomarket users' everyday lives undermined the capacity for many within the community to take real-world actions against drug prohibition. We argue that these tensions both within the individual and within the community, mobilised the community to internalise its politics to build the world they desired within the shell of the existing world. This tension remains today as the community that developed through the Silk Road continues to adapt and collaborate on iterations of cryptomarkets and tinker at the technical conditions for trust and anonymity. Because of this continued, yet adaptive, space within which the drug-use community seeks to develop a more permissive reality, the notion of constructive activism is likely to have continuing traction in revealing forms of collective activism online. 


\section{Acknowledgements}

We express heartfelt gratitude to the 17 Silk Road participants who shared their stories with us, and to the various community members who engaged with us and supported our work. We thank The Hub forum and the Dark Net Market Sub-Reddit for facilitating our work. We welcome your continued feedback on our ongoing work in this space.

The project was supported by internal funding from the National Drug Research Institute. The National Drug Research Institute in the Faculty of Health Sciences at Curtin University and the National Drug and Alcohol Research Centre at UNSW Australia are supported by funding from the Australian Government under the Substance Misuse Prevention and Service Improvement Grants Fund. M.B. is supported through a National Health \& Medical Research Council Early Career Researcher Fellowship (APP1070140), administered through UNSW Australia. The funders played no further part in the research process, and the views expressed in this paper should not be seen as representative of the views of the funders. 
References

Aldridge, J., \& Décary-Hétu, D. (2014). Not an 'Ebay for Drugs': The cryptomarket 'Silk Road' as a paradigm shifting criminal innovation. SSRN, http://ssrn.com/abstract=2436643.

Andersson, J. (2011). The origins and impacts of the Swedish file-sharing movement: A case study. Critical Studies in Peer Production (CSPP), 1(1), 1-18.

Barratt, M. J. (2012). Silk Road: eBay for drugs [letter to the editor]. Addiction, 107, 683. doi: 10.1111/j.1360-0443.2011.03709.x

Barratt, M. J., Allen, M., \& Lenton, S. (2014). 'PMA sounds fun': Negotiating drug discourses online. Substance Use and Misuse, 49(8), 987-998. doi: $10.3109 / 10826084.2013 .852584$

Barratt, M. J., Ferris, J. A., \& Winstock, A. R. (2014). Use of Silk Road, the online drug marketplace, in the UK, Australia and the USA. Addiction, 109(5), 774783. doi: $10.1111 /$ add.12470

Barratt, M. J., Lenton, S., \& Allen, M. (2013). Internet content regulation, public drug websites and the growth in hidden Internet services. Drugs: Education, Prevention, and Policy, 20, 195-202. doi: doi:10.3109/09687637.2012.745828

Barratt, M. J., \& Maddox, A. (forthcoming). Dark net ethnography: Conducting sensitive research in volatile digital spaces. Manuscript submitted for publication.

Bauman, Z., Bigo, D., Esteves, P., Guild, E., Jabri, V., Lyon, D., \& Walker, R. B. J. (2014). After Snowden: Rethinking the impact of surveillance. International Political Sociology, 8(2), 121-144. doi: 10.1111/ips.12048

Beer, D., \& Burrows, R. (2010). Consumption, prosumption and participatory web cultures. Journal of Consumer Culture, 10(1), 3-12. doi: $10.1177 / 1469540509354009$

Bennett, W. L., \& Segerberg, A. (2012). The logic of connective action. Information, Communication \& Society, 15(5), 739-768. doi: 10.1080/1369118x.2012.670661

Cardullo, P. (2015). 'Hacking multitude' and Big Data: Some insights from the Turkish 'digital coup'. Big Data \& Society, 2(1), 1-14. doi: $\underline{10.1177 / 2053951715580599}$

Chen, A. (2011, June 1). The underground website where you can buy any drug imaginable, Gawker. Available at: http://gawker.com/5805928/theunderground-website-where-you-can-buy-any-drug-imaginable (accessed 16 June 2011; archived at http://www.webcitation.org/5zTgI3sEH). 
Christin, N. (2013, May 13-17). Traveling the Silk Road: a measurement analysis of a large anonymous online marketplace. Paper presented at the International World Wide Web Conference (IW3C2), Rio de Janeiro, Brazil.

Coleman, E. G. (2010). The Hacker Conference: A ritual condensation and celebration of a lifeworld. Anthropological Quarterly, 83(1), 47-72.

Coleman, E. G. (2012). Coding freedom: The ethics and aesthetics of hacking. New Jersey: Princeton University Press.

Coleman, E. G. (2014). Hacker, hoaxer, whistleblower, spy: The many faces of Anonymous. London; New York: Verso.

Coleman, E. G., \& Golub, A. (2008). Hacker practice: Moral genres and the cultural articulation of liberalism. Anthropological Theory, 8(3), 255-277. doi: $10.1177 / 1463499608093814$

Federal Bureau of Investigation (FBI). (2013). Criminal complaint: Ross William Ulbricht a/k/a Dread Pirate Roberts, DPR, Silk Road. Available at: https://www.cs.columbia.edu/ smb/UlbrichtCriminalComplaint.pdf (accessed 30 November 2013; archived at http://www.webcitation.org/6LWf6foGa).

Garrett, K. (2006). Protest in an Information Society: A review of literature on social movements and new ICTs. Information, Communication \& Society, 9(2), 202224. doi: 10.1080/13691180600630773

Gehl, R. W. (2014). Power/freedom on the dark web: A digital ethnography of the Dark Web Social Network. New Media \& Society, Early View. doi: $10.1177 / 1461444814554900$

Gerbaudo, P., \& Treré, E. (2015). In search of the 'we' of social media activism: introduction to the special issue on social media and protest identities. Information, Communication \& Society, 18(8), 865-871. doi: 10.1080/1369118x.2015.1043319

Hine, C. (2000). Virtual ethnography. London: Sage.

Manion, M., \& Goodrum, A. (2000). Terrorism or civil disobedience: toward a hacktivist ethic. SIGCAS Computers and Society, 30(2), 14-19. doi: $10.1145 / 572230.572232$

Martin, J. (2014). Drugs on the dark net: How cryptomarkets are transforming the global trade in illicit drugs. New York: Palgrave Pivot.

McHugh, M. L. (2012). Interrater reliability: The kappa statistic. Biochemia Medica, 22(3), 276-282. doi:10.11613/BM.2012.031 
McInerney, P.-B. (2009). Technology movements and the politics of free/open source software. Science, Technology, \& Human Values, 34(2), 206-233. doi: $10.2307 / 29734084$

Ritzer, G., \& Jurgenson, N. (2010). Production, consumption, prosumption. Journal of Consumer Culture, 10(1), 13-36. doi: 10.1177/1469540509354673

Ulbricht, R. (2015) Letter to Judge Forrest, United States District Court of Southern District New York, United States of America v. Ulbricht. Case 1:14-cr-00068KBF Document 251-1 Filed 05/22/15 Page 1 of 3 Available at: https://s3.amazonaws.com/s3.documentcloud.org/documents/2086668/govuscourts-nysd-422824-251-1.pdf

van de Sande, M. (2015). Fighting with tools: Prefiguration and radical politics in the twenty-first century. Rethinking Marxism, 27(2), 177-194. doi: 10.1080/08935696.2015.1007791

van Hout, M. C., \& Bingham, T. (2013a). 'Silk Road', the virtual drug marketplace: A single case study of user experiences. International Journal of Drug Policy, 24, 385-391. doi: 10.1016/j.drugpo.2013.01.005

van Hout, M. C., \& Bingham, T. (2013b). 'Surfing the Silk Road': A study of users' experiences. International Journal of Drug Policy, 24, 524-529. doi: 10.1016/j.drugpo.2013.08.011

van Hout, M. C., \& Bingham, T. (2014). Responsible vendors, intelligent consumers: Silk Road, the online revolution in drug trading. International Journal of Drug Policy, 25, 183-189. doi: 10.1016/j.drugpo.2013.10.009 